\title{
The radial basis integral equation method for 2D Helmholtz problems
}

\author{
H. Dogan \& V. Popov \\ Wessex Institute of Technology, Environmental and Fluid Mechanics, \\ Southampton, UK
}

\begin{abstract}
A meshless method for the solution of 2D Helmholtz equation has been developed by using the Boundary Integral Equation (BIE) combined with Radial Basis Function (RBF) interpolations. BIE is applied by using the fundamental solution of the Helmholtz equation, therefore domain integrals are not encountered in the method. The method exploits the advantage of placing the source point always in the centre of circular sub-domains in order to avoid singular or near-singular integrals. Three equations for two-dimensional (2D) or four for three-dimensional (3D) potential problems are required at each node. The first equation is the integral equation arising from the application of the Green's identities and the remaining equations are the derivatives of the first equation in respect to space coordinates. RBF interpolation is applied in order to obtain the values of the field variable and partial derivatives at the boundary of the circular sub-domains, providing in this way the boundary conditions for solution of the integral equations at the nodes (centres of circles). The accuracy and robustness of the method has been tested on some analytical solutions of the problem. Two different RBFs are used, namely $f_{1}(R)=R^{2} \ln (R)+x+y+1$ and $f_{2}(R)=R^{4} \ln (R)+x^{2}+y^{2}+x y+x+1$. The latter has been found to produce more accurate results.

Keywords: meshless method, 2D Helmholtz equation, circular sub-domains, radial basis functions.
\end{abstract}

\section{Introduction}

The 2-D modelling of acoustics has been of significant importance for a variety of engineering problem in areas such as ultrasonics, microfluidics, aeoracoustics, etc. The numerical modelling of the problem is essential in case a complete 
theoretical approach is not possible, i.e. multiphase flows or in the absence of experimental research. Hence, robust numerical algorithms are needed. The robustness of the numerical scheme plays a more important role whilst dealing with high frequencies since the numerical pollution and dispersion affect the accuracy in a significant way [1]. Meshless methods have received attention in the last a few decades due to their several advantages over usual boundary element methods hence some approaches, using Galerkin method [2] and hybrid boundary node method [3], are present for solving the Helmholtz equation. In this report, we present a truly meshless Radial Basis Integral Equation Method (RBIEM) in order to solve the governing Helmholtz equation in a 2-D setting.

In the direct BEM formulation for the Helmholtz equation, difficulties in implementation arise due to singular integrals which require a special treatment [4], and furthermore the solution loses its accuracy significantly in the interior part of the domain close to the boundary which could be handled by integral transformations as [5] performed contour integration.

Similarly to the local boundary integral equation method [6], the RBIEM [7] implements the BEM equation over circular sub-domains where the source points are placed in the centres of the circles, which eliminates the above mentioned singular integrals. Three equations at each source point for $2 \mathrm{D}$ problems are solved, one for the field variable using the direct BEM formulation while the other two equations are the derivatives of the original equation in respect to spatial coordinates at the source point. The field variable and gradients over the circular boundaries are interpolated by using radial basis functions (RBFs). The augmented thin plate spline (ATPS) and fourth order ATPS with second order polynomial augmentation are used in this work. The fundamental solution, Hankel function of the second kind, of the interior 2D Helmholtz problem is used. The matrix coefficients resulting from the integration over the circular boundaries will be same provided that the radius remains the same, therefore they are evaluated only once.

The RBIEM differs from the LBIE in certain aspects [7]: (i) for the solution on the boundary, the LBIE replaces the circular domain with part of the global boundary and the remaining part of the circle, whereas the RBIEM keeps the circular integration approach on the. (ii) the LBIE uses the concept of "companion solution" in order to avoid the gradients/normal derivatives inside the problem domain while the RBIEM solves for the potential and the partial derivatives at each source node including the global boundary of the domain. (iii) The boundary conditions in the RBIEM are directly imposed at the source points on the global boundary, each replacing one of the three equations.

Since the integral domains are always circular, the integrals are regular regardless of the order of the derivative; though the integral kernels for the $2 \mathrm{D}$ Helmholtz equation include Hankel functions up to second order, whose evaluation is a challenging task $[4,5]$.

The method is briefly described in Sections 2 and 3, while numerical examples are presented in Section 4 with the conclusions in Section 5. The details concerning the solution procedures in the RBIEM can be found in [7]. 


\section{The radial basis integral equation method}

In this section the radial basis integral equation method (RBIEM) is introduced.

Let us consider the following Helmholtz equation:

$$
\left(\nabla^{2}+k^{2}\right) u(r)=0
$$

where $u(r)$ is a potential field, $r$ is a position vector. Given a point $r$ inside a domain $\Omega$, by applying the Green integral formula equation (1) can be transformed into the following integral form:

$$
\lambda(r) u(r)+\int_{\Gamma} q^{*}(r, \xi) u(\xi) d \Gamma_{\xi}-\int_{\Gamma} u^{*}(r, \xi) q(\xi) d \Gamma_{\xi}=0
$$

where $u^{*}(r, \xi)$ is the fundamental solution of the Helmholtz problem, $q(\xi)=\partial u(\xi) / \partial n$ and $q^{*}(r, \xi)=\partial u^{*}(r, \xi) / \partial n$. For a 2D problem the fundamental solution is given by

$$
\begin{gathered}
u^{*}(\mathrm{r}, \xi)=-\frac{i}{4} H_{0}^{2}(k R) \\
H_{0}^{2}(k R)=J_{0}(k R)-i Y_{0}(k R)
\end{gathered}
$$

where $J_{0}$ and $Y_{0}$ are Bessel functions of first and second kind, respectively and $R$ is the distance from the point of application of the concentrated unit source to any other point under consideration, i.e. $R=|r-\xi|$. The derivative of the fundamental solution could be evaluated analytically;

$$
q^{*}(\mathrm{r}, \xi)=\frac{\partial R}{\partial n} \frac{i k}{4} H_{1}^{2}(k R)
$$

The constant $\lambda(r)$ has value from 0 to 1 being equal to $1 / 2$ for smooth boundaries and 1 if the source point $r$ is inside the domain.

The proposed formulation solves in each interior node three integral equations in order to obtain the potential $u$, and the partial derivatives $\partial u / \partial x_{\mathrm{j}}$. Equation (6) is used to find the potential while the equations for derivatives $\partial u / \partial x_{\mathrm{j}}$ are obtained by differentiating (2) in respect to $x_{\mathrm{j}}$, where $x_{\mathrm{j}}$ are components of $r$. The derivatives of (2) are given below:

$$
\frac{\partial u(r)}{\partial x_{j}}=-\int_{\Gamma_{i}} \frac{\partial q^{*}(r, \xi)}{\partial x_{j}} u(\xi) d \Gamma_{\xi}+\int_{\Gamma_{i}} \frac{\partial u^{*}(r, \xi)}{\partial x_{j}} q(\xi) d \Gamma_{\xi}
$$

Note that the derivatives of the fundamental solution and its gradient appearing in the integral Kernels of Eqn. (6) can be evaluated analytically as given below: 


$$
\begin{gathered}
\frac{\partial u^{*}(r, \xi)}{\partial x_{j}}=\frac{\partial R}{\partial x_{j}} \frac{\partial u^{*}(r, \xi)}{\partial R}=\frac{\partial R}{\partial x_{j}} \frac{i k}{4} H_{1}^{2}(k R) \\
\frac{\partial q^{*}(r, \xi)}{\partial x_{j}}=\frac{\partial}{\partial x_{j}}\left\{\frac{\partial R}{\partial n} \frac{i k}{4} H_{1}^{2}(k R)\right\}=\frac{\partial^{2} R}{\partial x_{j} \partial n} \frac{i k}{4} H_{1}^{2}(k R)+\frac{\partial R}{\partial n} \frac{\partial R}{\partial x_{j}} \frac{\partial}{\partial R}\left\{\frac{i k}{4} H_{1}^{2}(k R)\right\} \\
=\frac{\partial^{2} R}{\partial x_{j} \partial n} \frac{i k}{4} H_{1}^{2}(k R)+\frac{\partial R}{\partial n} \frac{\partial R}{\partial x_{j}} \frac{i k}{4}\left\{k H_{2}^{2}(k R)-\frac{H_{1}^{2}(k R)}{R}\right\}
\end{gathered}
$$

The discretized form of (6) for the unknown $\partial u / \partial x_{\mathrm{j}}$ at node $i$ is given as:

$$
\frac{\partial u_{i}}{\partial x_{j}}=-\sum_{k=1}^{J_{b n}} \frac{\partial H_{i k}}{\partial x_{j}} u_{k}+\sum_{k=1}^{J_{b n}} \frac{\partial G_{i k}}{\partial x_{j}} q_{k}
$$

The normal derivative $q$ in (9) can be written as:

$$
q=\frac{\partial u}{\partial n}=\nabla u \cdot \vec{n}=\sum_{k} \frac{\partial u}{\partial x_{k}} n_{k}
$$

where $n_{k}$ are components of the unit normal vector. According to (10) equation (2) can be discretized as:

$$
u_{i}+\sum_{j=1}^{J} H_{i j} u_{j}-\sum_{m} \sum_{j=1}^{J} G_{i j m} \frac{\partial u_{j}}{\partial x_{m}}=0
$$

where $G_{\mathrm{ijm}}=G_{\mathrm{ij}} n_{\mathrm{m}}$. Substituting $q$ from (10) into (9) yields:

$$
\frac{\partial u_{i}}{\partial x_{j}}=-\sum_{n=1}^{J_{b n}} \frac{\partial H_{i n}}{\partial x_{j}} u_{n}+\sum_{p} \sum_{n=1}^{J_{b n}} \frac{\partial G_{i n p}}{\partial x_{j}} \frac{\partial u_{n}}{\partial x_{p}}
$$

where $G_{\text {inp }}=G_{\text {in }} n_{\mathrm{p}}$. Equation (11) can be written in matrix form as

$$
\mathbf{H u}-\sum_{m} \mathbf{G}_{m} \frac{\partial \mathbf{u}}{\partial x_{m}}=0
$$

and equations (12) can be written as:

$$
\frac{\partial \mathbf{u}}{\partial x_{j}}=-\frac{\partial \mathbf{H}}{\partial x_{j}} \mathbf{u}+\sum_{m} \frac{\partial \mathbf{G}_{m}}{\partial x_{j}} \mathbf{q}_{m}
$$

\section{Interpolation for the unknown values at the circular boundary of the sub-domain}

In order to perform the integration over the local boundaries of the circular subdomains, values of the potentials and partial derivatives must be known on the circles. In this work four quadratic continuous elements were employed to perform the integration over the circular boundaries. Eight fictitious nodes were 
introduced on the circular boundaries in order to define the four quadratic elements used in the integration over the circles. The values of the field variables at the eight nodes were determined through interpolation using the values of field variables at neighbouring nodes. The final system of equations solves for potentials and derivatives only at specified nodes for solution of the problem, which are located at centres of circular sub-domains, and not at the fictitious nodes on the circular boundaries. Only nodes at centres of sub-domains are used in the interpolation for obtaining the values of field variables at fictitious nodes on the circular boundaries. The unknown potential at one of the eight nodes, denoted by $\omega$ is approximated by $n$ neighbouring nodes $x_{\mathrm{i}}$ by the following formula:

$$
u(\omega)=\sum_{i=1}^{n} f\left(\omega, x_{i}\right) \cdot a_{i}
$$

The $n$ neighbouring nodes can be given as an input to the code, which would in part reduce the meshless nature of this approach, or can be generated automatically as was done in the present case. Two possibilities were considered: (i) to select the interpolation nodes by defining a suitable radius around $\omega$ which would contain the desired number of interpolation nodes, or (ii) to define the required number of interpolation nodes $n$ and let the numerical scheme find the nearest $\mathrm{n}$ nodes to $\omega$. In this case the option (ii) was adopted since it offered better control over the number of nodes used in the interpolation of the field variables.

Here $f$ is the ATPS function and $a_{\mathrm{i}}$ are the unknown coefficients. The unknown coefficients $a_{\mathrm{i}}$ are determined by constructing a system of equations which is obtained by applying (15) on neighbour nodal points $x_{\mathrm{i}}$ :

$$
u\left(x_{j}\right)=\sum_{i=1}^{n} f\left(x_{j}, x_{i}\right) \cdot a_{i}
$$

The following system of equations is formed

$$
\boldsymbol{u}_{0}=\boldsymbol{F}_{0} \boldsymbol{a}
$$

where $u_{0}=\left[u\left(x_{1}\right), u\left(x_{2}\right), \ldots, u\left(x_{n}\right)\right]^{\mathrm{T}}, F_{0}=f_{j i}=f\left(x_{j}, x_{i}\right), \quad j=1,2, \ldots, n ; i=1,2, \ldots, n$.

The unknown coefficients $a_{i}$ are determined by:

$$
\boldsymbol{a}=\boldsymbol{F}_{0}^{-1} \boldsymbol{u}_{0}
$$

The potential at point $\omega$ can be written as

$$
u(\omega)=F\left(\omega, x_{i}\right) F_{0}^{-1} u_{0}
$$

where $F\left(\omega, x_{i}\right)=\left[f\left(\omega, x_{1}\right), f\left(\omega, x_{2}\right), \ldots, f\left(\omega, x_{n}\right)\right]$.

The partial derivatives at $\omega$ are obtained as:

$$
\frac{\partial u(\omega)}{\partial x_{l}}=F\left(\omega, x_{i}\right) F_{0}^{-1} q_{0 l}
$$

where $q_{0 l}=\left[q_{l}\left(x_{1}\right), q_{l}\left(x_{2}\right), \ldots, q_{l}\left(x_{n}\right)\right]^{\mathrm{T}}$ and $q_{l}=\partial u / \partial x_{l}$.

Equation (20) produces more accurate approximation of the partial derivatives. 


\section{Numerical results}

\subsection{Solution of the helmholtz equation}

The Helmholtz Eqn. is used in order to verify the validity of the approach. A square domain with length $L=1$ at each side is considered. The $\partial \Phi / \partial x$ and $\partial \Phi / \partial y$ approach zero on the lines $\mathrm{x}=0$ and $\mathrm{y}=0$, therefore the domain is shifted $(0.5,0.5)$ from the origin for a better visualisation of relative error. The following boundary conditions are applied:

$$
\begin{gathered}
\left.\Phi\right|_{x=1.5}=\mathrm{J}_{0}(\mathrm{kr})+\mathrm{i}(\cos (1.5 \mathrm{k})+\sin (\mathrm{ky})) \\
\left.\Phi\right|_{y=1.5}=\mathrm{J}_{0}(\mathrm{kr})+\mathrm{i}(\cos (\mathrm{kx})+\sin (1.5 \mathrm{k})) \\
\left.\frac{\partial \Phi}{\partial n}\right|_{x=0.5}=\frac{k}{2} \mathrm{~J}_{1}(\mathrm{kr}) / \mathrm{r}+\mathrm{i} \mathrm{k} \sin \left(\frac{\mathrm{k}}{2}\right) \\
\left.\frac{\partial \Phi}{\partial n}\right|_{y=0.5}=\frac{k}{2} \mathrm{~J}_{1}(\mathrm{kr}) / \mathrm{r}-\mathrm{i} \mathrm{k} \cos \left(\frac{\mathrm{k}}{2}\right)
\end{gathered}
$$

which yield the solution $\Phi=\mathrm{J}_{0}(\mathrm{kr})+\mathrm{i}(\cos (\mathrm{kx})+\sin (\mathrm{ky}))$.

The ATPS is used in all of the RBF computations. The built-in Fortran functions getri and solver pardiso are used to compute the inverse matrix formed in RBF interpolation and to solve the overall sparse system, respectively. The models of sizes up to 361201 source nodes in the domain with uniform distribution, equivalent to $1,083,603$ degrees of freedom (DOF), have been solved on a workstation Intel Xeon $3.2 \mathrm{GHz}$.

\subsubsection{The parameters that affect the efficiency of the method}

In this section, we investigate the parameters that affect the efficiency and accuracy of the algorithm. Although the results obtained for $\Phi=\mathrm{J}_{0}(\mathrm{kr})+\mathrm{i}(\cos (\mathrm{kx})+\sin (\mathrm{ky}))$ are presented here, these optimal values show the same behaviour when tested individually with the functions $\operatorname{Re}(\Phi)$ and $\operatorname{Im}(\Phi)$. The effect of two major components: (i) the RBF approximation and (ii) circular integration around nodes, on the numerical error is analyzed. Keeping the number of the nodes used in the computations sufficiently large, numerical experiments were performed in order to determine more optimal values for each of the parameters. As the base case values we set Nodes $=10201, \mathrm{~N}_{\omega}=24, \mathrm{~N}_{\mathrm{d}}=16, \mathrm{~N}_{\mathrm{b}}=12, R_{d}=D_{x}$ and $R_{b}=D_{x}$. The results are displayed by examining the L2-norms for the components $\operatorname{Re}(\Phi), \operatorname{Re}(\partial \Phi / \partial x)$, $\operatorname{Re}(\partial \Phi / \partial y), \operatorname{Im}(\Phi), \operatorname{Im}(\partial \Phi / \partial x)$ and $\operatorname{Im}(\partial \Phi / \partial y)$, where $k$ is wavenumber; $\lambda$ is wavelength; $N_{\omega}$ is the number of fictitious points used in the circular integration around each node; $R_{d}$ is the radius of the circular sub-domain in the 
domain; $R_{b}$ is the radius of the circular sub-domain on the boundary; $\mathrm{N}_{\mathrm{d}}$ is the number of points used in RBF interpolation in the domain; $\mathrm{N}_{\mathrm{b}}$ is the number of points used in RBF interpolation on the boundary; $D_{x}$ is the distance between nodes in $x$ and $y$ directions in case of uniform distribution.

The L2-norm error for $\Phi$ is defined as

$$
e_{L 2}=\sqrt{\frac{\sum_{i=1}^{N}\left(\Phi_{i}-\Phi_{i}^{n}\right)^{2}}{\sum_{i=1}^{N} \Phi_{i}{ }^{2}}} 100 \%
$$

where $\mathrm{N}$ is the number of nodes, $\Phi_{i}^{n}$ the potential at node $i$ by numerical method and $\Phi_{\mathrm{i}}$ the analytical solution at node $i$.

Firstly, we investigated the parameters related to the circular integration around nodes. Tests were carried out in order to determine the optimal integration radius $R$ by prescribing analytical solution values of both potential and the derivatives on the circular boundary. We conclude that a ratio $R_{d} / \lambda \leq$ 0.01 is required for accurate results in the computations. Next, the influence of ratio $R_{d} / D_{x}$ on the accuracy of the solution has been investigated. The numerical experiments suggested a value of $R_{d} / D_{x}=1$ as one leading to accurate results, as can be seen in Figure 1.

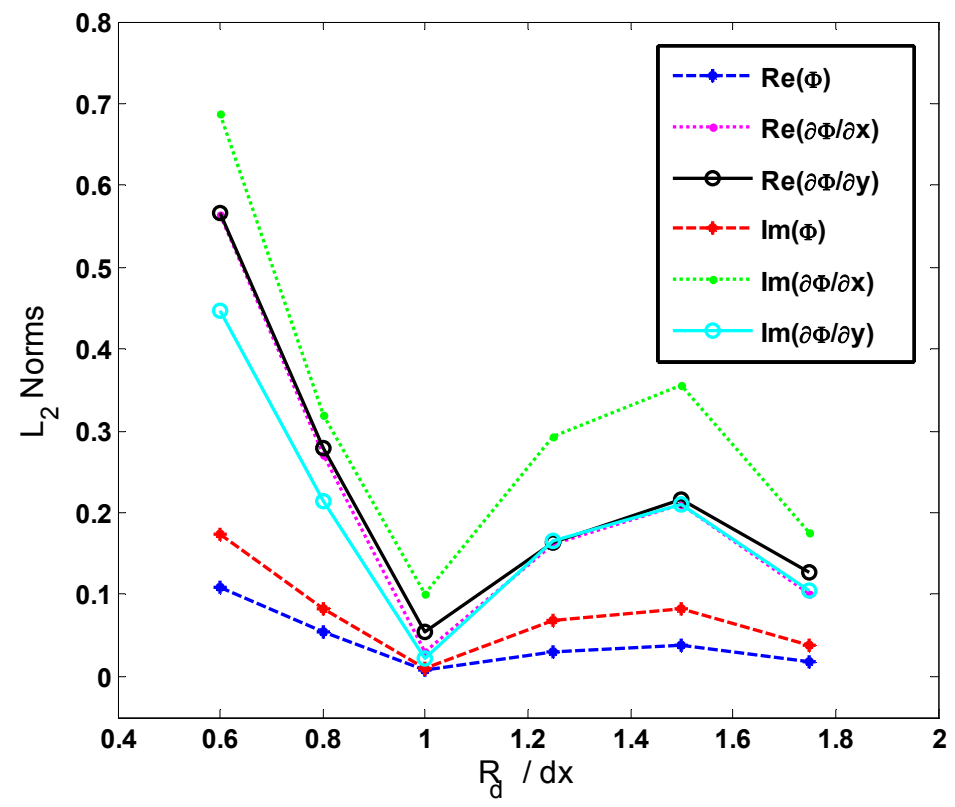

Figure 1: L2 Norm error for different $R_{d} / D_{x}$ with Nodes $=10201, \mathrm{~N}_{\omega}=24$,

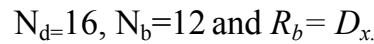


The number of neighbouring interpolation points used in the RBF has been increased up to 48 , which is sufficiently large. The results show slight improvement up to this point, whereas numerical error dominates afterwards. The results reach a nearly stable behaviour for $\mathrm{N}_{\mathrm{d}} \geq 28$ (Fig. 2). Nevertheless, a choice of 16 gives the best accuracy which is also preferable to the large numbers due to the significant reduction in the sparsity of the overall matrix and computation time in that regard.

The behaviour of the method is investigated near the boundaries by varying the integration radius $R_{b}$ and the number of nodes in the RBF for the nodes on the boundary, $\mathrm{N}_{\mathrm{b}}$, close to the boundary or far inside the domain. Note that keeping the same value would decrease the computation time since the circular integration results in the same coefficients for fixed radius and number of boundary elements used. Reducing the $\mathrm{R}_{\mathrm{b}}$ and $\mathrm{N}_{\mathrm{b}}$ show slightly more accurate results, hence related figures are not shown here.

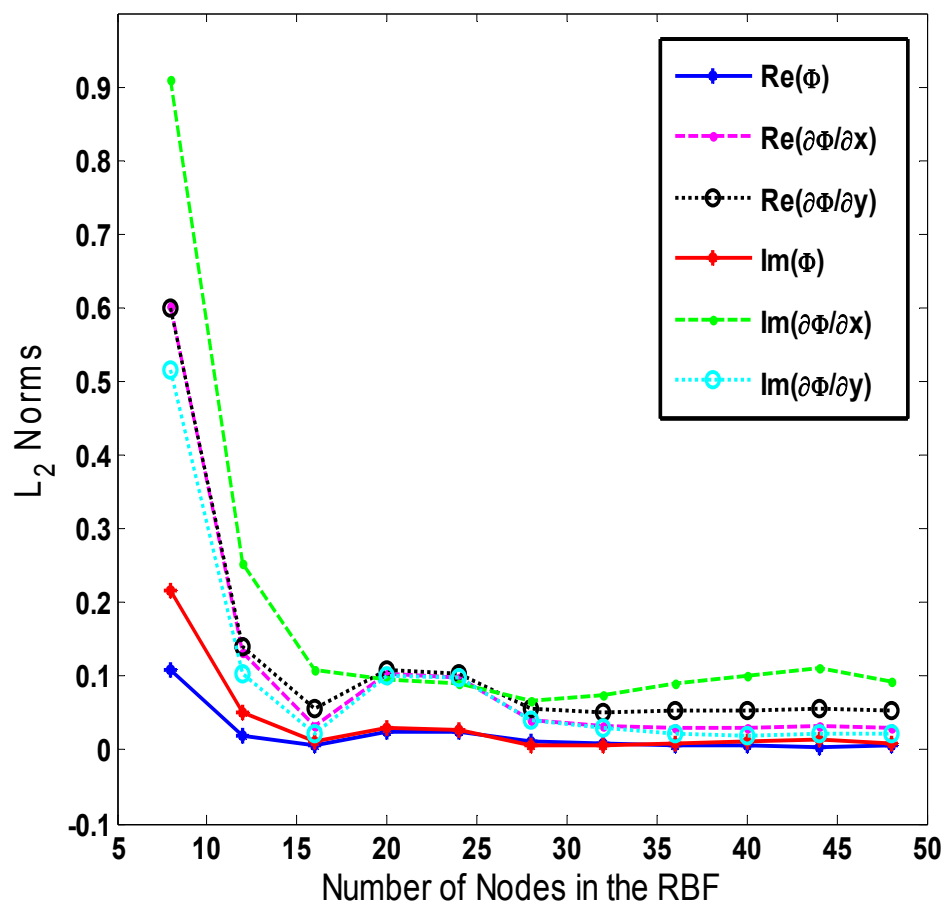

Figure 2: L2 Norm error for different $\mathrm{N}_{\mathrm{d}}$ with Nodes $=10201, \mathrm{~N}_{\omega}=24, R_{d}=D_{x}$ and $R_{b}=D_{x}$.

The overall convergence of the method is stated clearly by the Figure 3 where the computations are carried with up to 360201 nodes. 


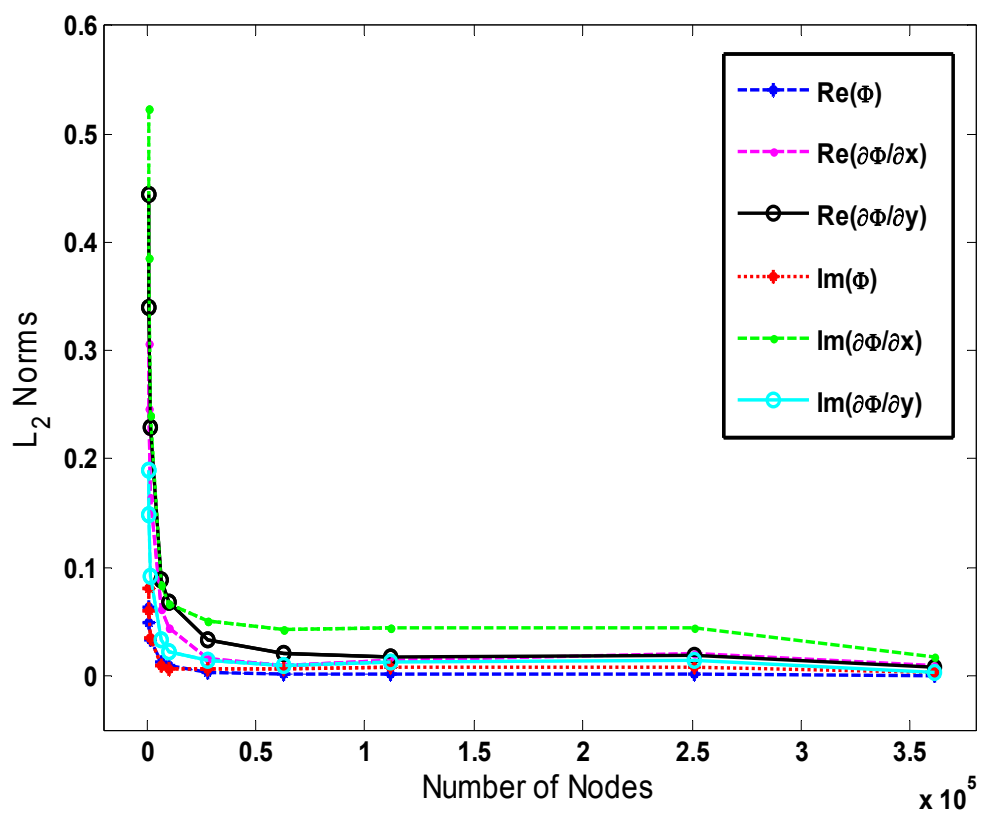

Figure 3: L2 Norm convergence with increasing the number of Nodes,

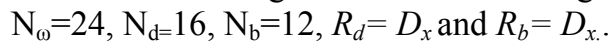

\subsection{Example 2}

Another solution to the Helmholtz equation is used in order to test the effect of the RBFs. A square domain with length $L=1$ at each side is considered. The following boundary conditions are applied:

$$
\begin{gathered}
\left.\Phi\right|_{y=0.5}=\operatorname{Sin}\left(\frac{\sqrt{2}}{2} k x\right) \operatorname{Sin}\left(\frac{\sqrt{2}}{4} k\right) \\
\left.\Phi\right|_{y=-0.5}=\operatorname{Sin}\left(\frac{\sqrt{2}}{2} k x\right) \operatorname{Sin}\left(-\frac{\sqrt{2}}{4} k\right) \\
\left.\frac{\partial \Phi}{\partial n}\right|_{x=0.5}=k \operatorname{Cos}\left(\frac{\sqrt{2}}{4} k\right) \operatorname{Sin}\left(\frac{\sqrt{2}}{2} k y\right) \\
\left.\frac{\partial \Phi}{\partial n}\right|_{x=-0.5}=k \operatorname{Cos}\left(-\frac{\sqrt{2}}{4} k\right) \operatorname{Sin}\left(\frac{\sqrt{2}}{2} k y\right)
\end{gathered}
$$


which yield the solution $\Phi=\operatorname{Sin}\left(\frac{\sqrt{2}}{2} k x\right) \operatorname{Sin}\left(\frac{\sqrt{2}}{2} k y\right)$.

Two different RBFs are used:

$$
\begin{gathered}
\text { (i) } f_{1}(R)=R^{2} \ln (R)+x+y+1 \\
\text { (ii) } f_{2}(R)=R^{4} \ln (R)+x^{2}+y^{2}+x y+x+1
\end{gathered}
$$

The tests related to the best accuracy of the method were performed for the second RBF, $f_{2}$, as well. The optimum parameters were found to be the same with the first RBF except the number of fictitious nodes on each circle of integration, e.g. $\mathrm{N}_{\omega}=24, \mathrm{~N}_{\mathrm{d}=16}, \mathrm{~N}_{\mathrm{b}}=12$ and $R_{b}=D_{x}$. For the sake of brevity, related figures are not re-plotted here. As seen in Table 1, the solution converges

Table 1: $\quad$ L2 Norm error comparison for $f_{l}$ and $f_{2}$ RBFs with $k=6$.

\begin{tabular}{|c|c|c|}
\hline$f_{1}$ & $f_{2}$ & \\
\hline $\begin{array}{c}\boldsymbol{R e}(\boldsymbol{\Phi}) \\
\text { L2 Norm }\end{array}$ & $\begin{array}{c}\boldsymbol{R e}(\boldsymbol{\Phi}) \\
\text { L2 Norm }\end{array}$ & $\begin{array}{c}\text { Number of } \\
\text { Nodes }\end{array}$ \\
\hline 2.4937 & 0.1624 & 441 \\
\hline 0.7906 & 0.0348 & 1681 \\
\hline 0.2887 & 0.0024 & 6561 \\
\hline 0.1849 & 0.0033 & 10201 \\
\hline 0.0237 & 0.0071 & 40401 \\
\hline 0.0149 & 0.0075 & 63001 \\
\hline 0.0280 & 0.0081 & 160801 \\
\hline 0.0624 & 0.0078 & 251001 \\
\hline
\end{tabular}

Table 2: $\quad$ L2 Norm error for $f_{2}$ RBFs with high wavenumbers.

\begin{tabular}{|c|c|c|c|c|}
\hline$k$ & $\begin{array}{c}\operatorname{Re}(\Phi) \\
\text { (L2 Norm) }\end{array}$ & $\begin{array}{c}\operatorname{Re}(\partial \Phi / \partial x) \\
\text { (L2 Norm) }\end{array}$ & $\begin{array}{c}\operatorname{Re}(\partial \Phi / \partial x) \\
\text { (L2 Norm) }\end{array}$ & Nodes \\
\hline 20 & 0.0156 & 0.0242 & 0.0207 & 63001 \\
\hline 36.2 & 0.0557 & 0.0489 & 0.0536 & 251001 \\
\hline 43.18 & 0.0543 & 0.0515 & 0.0480 & 361201 \\
\hline 50.02 & 0.0457 & 0.0541 & 0.0506 & 361201 \\
\hline 61.36 & 0.0573 & 0.0554 & 0.0543 & 361201 \\
\hline 72.05 & 0.0962 & 0.0989 & 0.1039 & 361201 \\
\hline 78.29 & 0.2180 & 0.2163 & 0.2156 & 361201 \\
\hline
\end{tabular}


more rapidly using $f_{2}$ in comparison to $f_{1}$. Therefore, the usage of $f_{2}$ allows us to solve the equation with higher wavenumbers. In Table 1, we can also notice the phenomena related to the optimum choice of $R_{d} / \lambda$, namely a further increase of the source nodes after an accurate solution is reached yields the so called pollution effect. Finally in Table 2, the results are provided with the high wavenumbers for the analytical solution $\operatorname{Sin}((\sqrt{2} / 2) k x) \operatorname{Sin}((\sqrt{2} / 2) k y)$. The results are acceptable up to $k=78.29$. The resonant frequencies were avoided as much as possible while choosing the wavenumbers.

\section{Conclusions}

A meshless method for the solution of 2D Helmholtz equation has been developed by using the Boundary Integral Equation (BIE) combined with Radial Basis Function (RBF) interpolations. BIE is applied by using the fundamental solution of the Helmholtz equation, therefore domain integrals are not encountered in the method. The method exploits the advantage of placing the source point always in the centre of circular sub-domains in order to avoid singular or near-singular integrals. RBF interpolation is applied in order to obtain the values of the field variable and partial derivatives at the boundary of the circular sub-domains, providing this way the boundary conditions for solution of the integral equations at the nodes (centres of circles). The accuracy, robustness and efficiency of the method have been validated on some analytical solutions of the problem. The parameters emerging from the BIE and RBF, such as radius of circular integration, number of neighbouring nodes in the RBF interpolation, choice of RBF etc., have been tested to certain extent to determine more optimal values which yield to more accurate solutions. In the placement of source nodes, a distance of 0.01-0.02 of the wavelength between the nodes is essential to catch the wave behaviour precisely. An integration radius equal to this distance is found to give more accurate results. Among the two RBFs used, $f_{2}(R)=R^{4} \ln (R)+x^{2}+y^{2}+x y+x+1$ has shown more rapid convergence and accuracy than $f_{1}(R)=R^{2} \ln (R)+x+y+1$.

\section{Acknowledgement}

The present study was supported by the SONO project, contract number: 228730 , as part of the Seventh Framework Programme (FP7-NMP-2008-Large-2).

\section{References}

[1] Wenderodt C, v. Estorff, O. Dispersion analysis of the meshfree radial point interpolation method for the Helmholtz equation. Int. J. For Numer. Methods In Engineering 2009; 77: 1670-1689. 
[2] Bouillar Ph., Suleau S. Element-Free Galerkin solutions for Helmholtz problems: formulation and numerical assessment of the pollution effect. Compu. Methods Appl. Mech. Eng. 1998; 162: 317-335.

[3] Miao Y., Wang Y., Wang Y. H. A meshless hybrid boundary-node method for Helmholtz problems. /Engineering Analysis with Boundary Elements/ 2009; 33: 120-127.

[4] Tadeu A. J. B., Santos P. F. A., Kausel E. Closed-form integration of singular terms for constant, linear and quadratic boundary elements. Part 2. SV-P wave propagation. Engineering Analysis with Boundary Elements 1999; 23: 757-768.

[5] Elliot D., Johnston P. R. Gauss-Legendre quadrature for the evaluation of integrals involving the Hankel function. Jour. Of Computational and Applied Mathematics 2008; 211: 23-35.

[6] Zhu T, Zhang J-D, Atluri S.N. A local boundary integral equation (LBIE) method in computational mechanics, and a meshless discretization approach. Computational Mechanics 1988; 21: 223-235.

[7] Popov V., Bui T. T. A meshless solution to two-dimensional convectiondiffusion problems. Engineering Analysis with Boundary Elements, 2010; 34; 680-689. 